\title{
Journal of the International AIDS

\section{Evaluation of kidney toxicity in HIV patients with tenofovir-based regimen: the role of boosted protease inhibitor in real clinical setting}

\author{
F Ortu* , P Piano, P Serra, R Meleddu, N Corso and PE Manconi
}

Address: Azienda Ospedaliero Universitaria di Cagliari, Monserrato, Italy

* Corresponding author

from Ninth International Congress on Drug Therapy in HIV Infection

Glasgow, UK. 9-13 November 2008

Published: 10 November 2008

Journal of the International AIDS Society 2008, I I (SuppI I):PI33 doi:I0.II86/I758-2652-I I-SI-PI33

This abstract is available from: http://www.jiasociety.org/content/II/SI/PI33

(C) 2008 Ortu et al; licensee BioMed Central Ltd.

\section{Purpose of the study}

Tenofovir (TFV) disoproxil fumarate (TDF) is an oral prodrug of the nucleotide reverse transcriptase inhibitor TFV. Following oral administration, TDF undergoes rapid conversion to TFV in plasma. TFV is eliminated from systemic circulation renally through a combination of glomerular filtration and active tubular secretion. Active tubular secretion of TFV has been inferred from the observation that TFV renal clearance exceeds that of creatinine clearance in patients. Renal drug-drug interactions may occur between therapeutics that are substrates for the same tubular transport pathways or that inhibit the pathway of a drug subject to renal excretion: both of them, ritonavir (RTV) and tenofovir, share the same transport pathways (MRP4) [Ray et al. Antim Ag Chem 2006 (Oct); 50: 32973304]. The objective of the present study was to investigate in a real clinical setting the relationship between $\mathrm{PI} / \mathrm{r}$ co-administration with TDF and changes in estimated renal function.

\section{Methods}

Our study involved 187 patients in TFV-based regimen: 49 in NNRTI and 138 with PI/r. In a follow-up of one year, we evaluated renal function by calculated glomerular filtrate using the Cockcroft-Gault (C-G) equation and the unabbreviated Modification of Diet in Renal Disease (MDRD) equation.

\section{Results}

The mean baseline renal function was within the normal range and was similar between treatment groups. In univariate analysis, decreases in $\mathrm{C}-\mathrm{G}$ estimates of $\mathrm{CrCl}$ were not significantly different among the two groups during the first 12 months of therapy.

\section{Conclusion}

In conclusion, our study demonstrated that also in reallife conditions, patients receiving TDF in combination with $\mathrm{PI} / \mathrm{r}$-based regimens had greater declines in renal function than did TDF NNRTI. 